\title{
Nuclídeos cosmogênicos: uma análise sobre um século de pesquisa em Geociências
}

\author{
COSMOGENIC NUCLIDES: AN ANALYSIS OF A CENTURY OF RESEARCH IN GEOSCIENCES.
}

\author{
Cristiane Heredia Gomes ${ }^{1 *}$, Diogo Gabriel Sperandi0 ${ }^{1}$ e Rafael Lima Dessart ${ }^{2}$ \\ 1-Univ. Fed. do Pampa, UnipamPa, Caçapava do Sul, RS, Brasil. emall: *cristianegomes@unipampa.edu.br; gabrielspe@gmall.com \\ 2-Univ. Fed. do Paraná, UfPR, Curitiba, PR, Brasil. emall: rldessrt@gmall.com
}

Abstract: The Portuguese language with more than 20 years of research in Cosmogenic Nuclides, still lacks literature and bibliographies on this subject, compared to English speaking contries. In Brazil, studies dedicated to research in this area are rare and scarce. Thus, Cosmochronology in Brazilian Science is new and mostly unknown. In order to promote the discussion about the applications of cosmogenic nuclides in science and its history, even if in a small way, we present this paper. It seeks to carry out a historical reconstruction of the research on cosmogenic nuclides and their important implications in Geosciences. Cosmogenic nuclides represent an indispensable tool for the study, understanding and determination of variational rates as a function of time. Therefore, in the last decades, the application of these isotopes in the most diverse geological environments, such as coastal, lacustrine, glacial, volcanic, tectonic, and extraterrestrial environments, has been explored and embraced within Geology.

\section{Manuscrito:}

Recebido: 10/02/18

Corrigido: $18 / 06 / 18$

Aceito: $28 / 06 / 18$

Citation: Gomes C.H., Sperandio D.G., Dessart R.L. 2018. Nuclídeos cosmogênicos: uma análise sobre um século de pesquisa em geociências. Terræ Didatica, 14(3):207-216. URL: http://www. ige.unicamp.br/terraedidatica/.

Keywords: Geosciences, Cosmogenic particles, chlorine, beryl, aluminum.

\section{Introdução}

Os produtos de reações de raios cósmicos são conhecidos como nuclídeos cosmogênicos (Granger \& Riebe 2014). Os nuclídeos cosmogênicos são uma importante ferramenta para estimar e determinar de forma direta o tempo e as taxas em que determinados processos atuam na superfície terrestre de forma a modificá-la. Nuclídeos Cosmogênicos têm sua origem a partir do bombardeio de raios cósmicos e a ação direta deste sobre a superfície terrestre. Estima-se que $\sim 10^{2}-10^{3}$ nuclídeos cosmogênicos in situ são gerados por grama de rocha por ano na superfície do solo (Lal \& Peter 1967). Estudos envolvendo nuclídeos cosmogênicos tornam possível a reconstrução e compreensão de movimentos tectônicos pré-históricos (Paleotectônica e Paleogeografia), na quantificação das taxas de denudação e erosão para diferentes intervalos cronológicos em variados ambientes geológicos (glacial, fluvial, eólico, costeiro, cárstico e tantos outros). Pode-se obter um detalhamento geológico recente do desenvolvimento geomorfológico não obtido com as técnicas analíticas convencionais. Estes nuclídeos são utilizados nos estudos de sistemas hidrográficos, que sejam ou não propulsores de desastres naturais (Williams et al. 2005), bem como, apresentam aplicação na composição de idades de soterramento.
A utilização de nuclídeos cosmogênicos para determinação de taxas de variação ao longo do tempo nos permite dizer (e compreender) o quanto a geomorfologia de determinada região mudou, ou então o quão rápido um rio pode mudar o seu curso, assim como, verificar os processos de mudanças climáticas registradas no aumento ou na diminuição de geleiras (Von Blanckenburg \& Willenbring 2014).

Pesquisas científicas de petróleo ou seus derivados foram intensificadas nas últimas décadas em diversas partes do mundo através do uso de nuclídeos cosmogênicos. A busca por este recurso finito e não renovável é uma rotina conhecida das indústrias petrolíferas. Talvez grande parte dos reservatórios já tenha sido descoberta devido ao aumento constante das investigações, causada em função do aumento na demanda deste recurso.

A acumulação de nuclídeos cosmogênicos em minerais ou na superfície de rochas vem sendo vista como a base para a datação de superfícies expostas e a quantificação de taxas de denudação, erosão e exumação (Gosse \& Phillips 2001, Granger \& Riebe 2014. Fig. 1), em idades de soterramento (Laureano et al. 2014) e em estudos voltados à paleoclimatologia (Balco \& Stone 2005). Embora para este fim o estudo de nuclídeos cosmogênicos seja recente, o princípio físico é conhecido a mais de um século.

\begin{tabular}{c|c|c|c|c|c}
\hline (C) Terrae Didat. & Campinas, SP & v.14 & n.3 & p. 207-216 & jul./set. 2018 \\
\hline
\end{tabular}




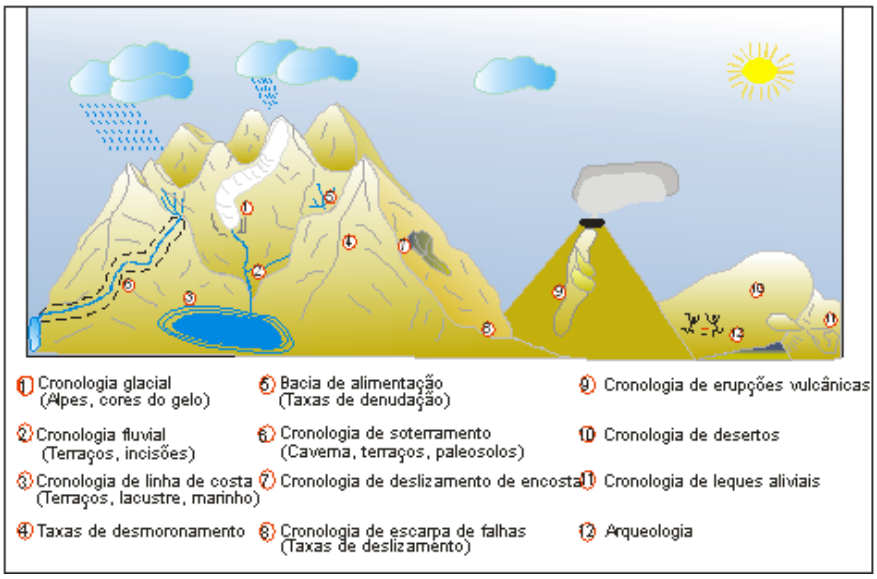

Figura 1. Ilustração de alguns exemplos de aplicação de estudos da técnica de datação com nuclídeos cosmogênicos em Geociências (Gosse \& Phillips 2001)

Os nuclídeos cosmogênicos terrestres são produzidos na superfície das rochas pela interação de raios cósmicos secundários com o núcleo dos átomos presentes em minerais, tais como: feldspato, olivina e quartzo (Gosse \& Phillips 2001). A formação de nuclídeos cosmogênicos é possível porque prótons de radiação cósmica colidem com partículas da atmosfera e geram nêutrons secundá- rendeu à Hess, o prêmio Nobel de Física em 1936.

Cerca de 22 anos depois da descoberta de Hess, a técnica de datação e modelamento com nuclídeos cosmogênicos teve início. Grosse (1934), em seus trabalhos experimentais foi o primeiro a sugerir que a radiação cósmica poderia produzir nuclídeos cosmogênicos em materiais da superfície terrestre.

Cinco anos mais tarde, após a descoberta de nêutrons em raios cósmicos (Locker 1933), os estudos de Montgomery e Montgomery (1939) apresentaram a possibilidade de produção de ${ }^{14} \mathrm{C}$ (radiocarbono) na atmosfera terrestre pela radiação cósmica. A procura inicial por radiocarbono na natureza foi realizada por Libby et al. (1946), que culminou na detecção de ${ }^{14} \mathrm{C}$ na natureza por Anderson et al. (1947). Pouco depois, Arnold e Libby (1949) demonstraram a viabilidade do uso de ${ }^{14} \mathrm{C}$ cosmogênico na datação arqueológica, isto é, na datação de materiais de origem humana. Isto marcou o inicio das pesquisas para as mudanças isotópicas produzidas por raios cósmicos em amostras terrestres e rios (Figura 2). Desta maneira, os nuclídeos cosmogênicos podem apresentar-se tanto de forma estável $\left({ }^{3} \mathrm{He},{ }^{21} \mathrm{Ne}\right)$ quanto na forma radioativa $\left({ }^{10} \mathrm{Be},{ }^{14} \mathrm{C},{ }^{26} \mathrm{Al} \mathrm{e}{ }^{36} \mathrm{Cl}\right)$.

\section{Nuclídeos Cosmogênicos}

Em 1912, o cientista austríaco Victor Franz Hess, realizando estudos na atmosfera terrestre com um eletroscópio associado a um detector de ionização (Bustamante 2013), descobriu que em altas altitudes o ar era até três vezes mais ionizado do que ao nível do mar (Figura 3). Deste modo, o cientista percebeu que a fonte de radiação ionizante não poderia estar na atmosfera e tampouco na Terra, mas sim, era vinda de fora e penetrava a atmosfera. Descobriu-se assim, a radiação cósmica. Tal feito

\section{Produção de particulas secundárias na atmosfera e nas rochas.} compilado de Gosse \& Phillips, 2001.

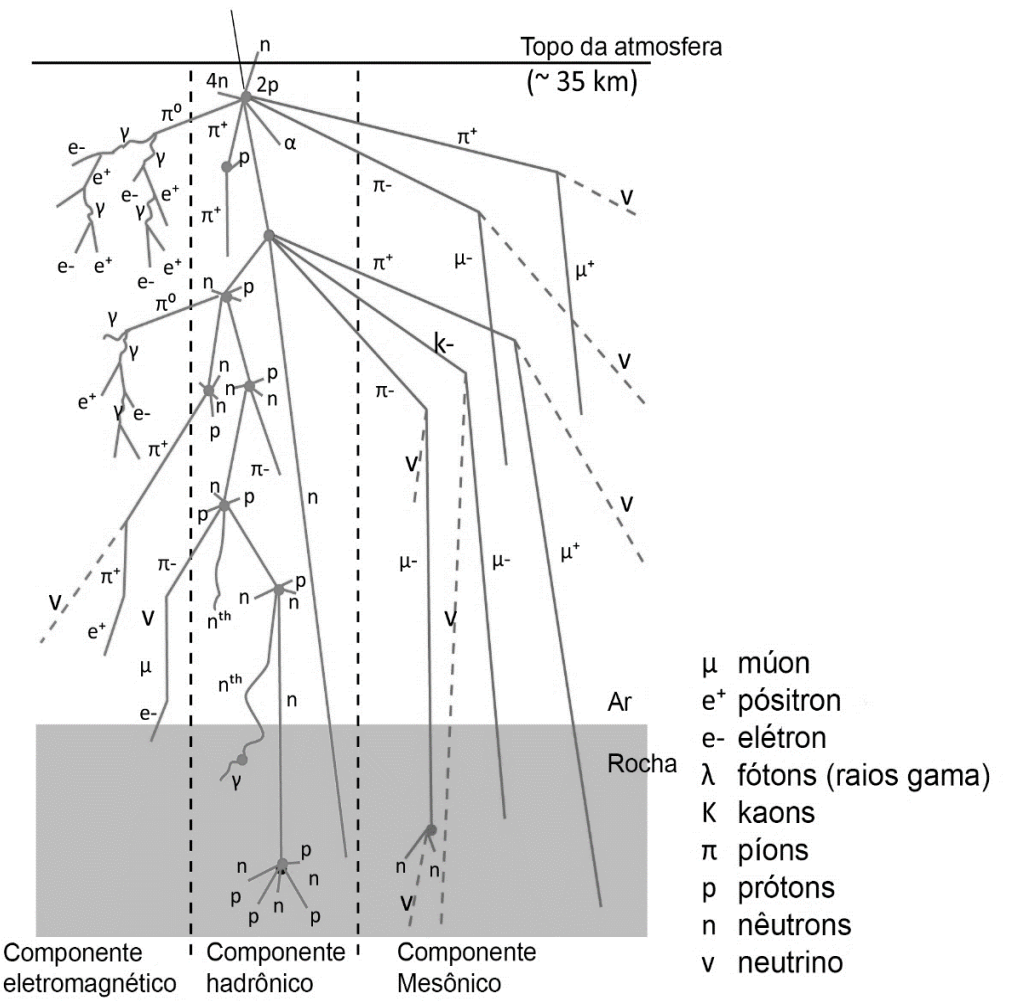

Figura 2. Produção de partículas secundárias na atmosfera e nas rochas (Gosse \& Phillips 2001) 


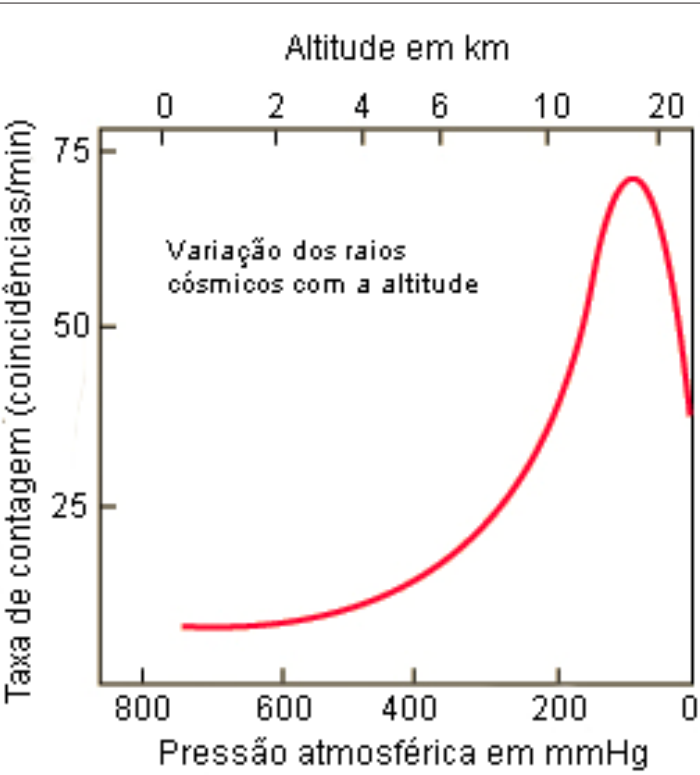

Figura 3. Taxa de contagem e pressão atmosférica, demonstrando a variação dos raios cósmicos frente à variação da altitude. Evidencia-se o aumento da ionização em altas altitudes (Nave 2005)

extra-terrestres, abrindo uma nova possibilidade de aplicação nas Geociências, principalmente no estudo de esqueletos, dentes, conchas e demais materiais de origem orgânica.

A produção do ${ }^{14} \mathrm{C}$ ocorre quando raios cósmicos bombardeiam o nitrogênio $\left({ }^{14} \mathrm{~N}\right)$, o qual irá absorver um nêutron e liberar um próton. Dando assim, origem ao carbono $\left({ }^{14} \mathrm{C}\right)$ e iniciando, então, o ciclo do carbono nos organismos vivos (Nave 2005. Fig. 4).

A partir disto, tendo a ciência dado os primeiros passos em direção ao avanço da cosmoquímica com os estudos de Locker (1933), Grosse (1934), Montgomery \& Montgomery (1939), Libby et al. (1946), Anderson et al. (1947) e Arnold \& Libby (1949), influenciados também pelos estudos de Hess (1912), dezenas de nuclídeos cosmogênicos estáveis e instáveis foram descobertos.

Davis \& Schaffer (1955) sugeriram que nuclídeos cosmogênicos poderiam ser produzidos pelo bombardeio de raios cósmicos nos minerais constituintes de rochas expostas na superfície terrestre. Assim, a fim de verificar esta suposição, eles mediram a concentração do nuclídeo cosmogênico in situ de ${ }^{36} \mathrm{Cl}$ em uma amostra de rocha máfica, utilizando uma metodologia semelhante a empregada para a datação com ${ }^{14} \mathrm{C}$. Eles demonstraram que os nuclídeos cosmogênicos de ${ }^{36} \mathrm{Cl}$ poderiam estimar as idades de rochas expostas em superfícies, mesmo que essas estivessem desniveladas e com relevos acentuados.
Até meados dos anos 50, poucos eram os estudos aplicados a cosmocronologia. Dentre os diversos fatores que dificultavam os avanços científicos na época, um deles era a análise de concentrações muito baixas de nuclídeos com vida suficientemente longa, como por exemplo, para o isótopo de alumínio $\left({ }^{26} \mathrm{Al}\right)$ com uma meia-vida de $7,4 \times 10^{5}$ anos.

Entre os anos de 1950 e 1960 houve o desenvolvimento de modelos para descrever a interação entre a radiação cósmica, o campo geomagnético e a atmosfera. Dados sobre as desintegrações nucleares na estratosfera e troposfera foram sintetizados por Lal \& Peters (1967), que projetaram o modelo global da produção estelar utilizado até os dias de hoje. O procedimento utilizado por Lal e colaboradores é bastante preciso na variação da taxa de produção com relação à altitude e latitude na atmosfera. As taxas de produção calculadas por eles dos nuclídeos na troposfera e na estratosfera, que corresponde à intensidade de raio cósmico observada durante 1948-49, foi determinada.

Em 1969, com a chegada do homem à lua (em 16 de julho daquele ano) tornou-se possível a realização de estudos sobre interação dos raios cósmicos nas rochas lunares (Reeves et al. 1970; Stoenner et al. 1970). Esta fase foi conhecida como a era da lua, onde as pesquisas relacionadas com o espaço eram muito populares e bem financiadas, pois até então, só meteoritos haviam sido analisados. Neste

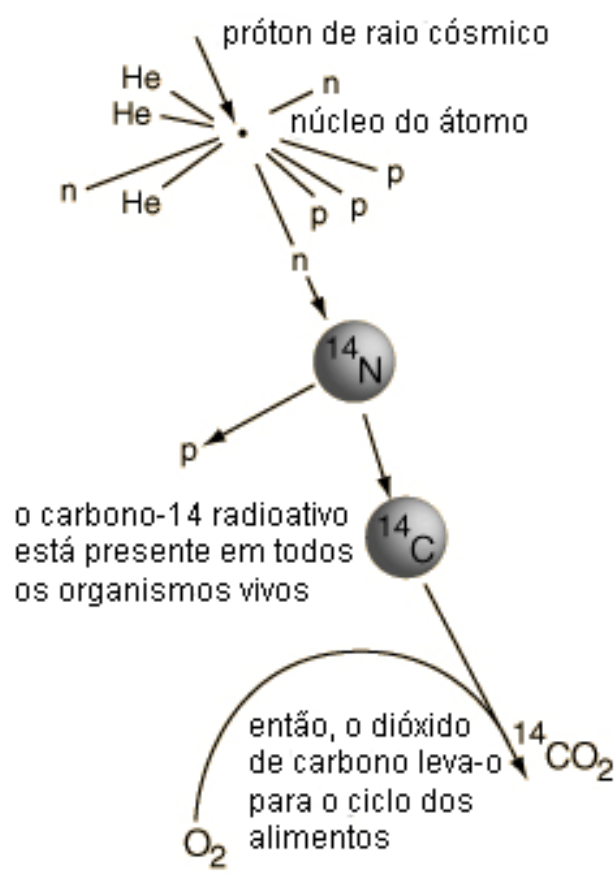

Figura 4. (a) Produção de ${ }^{14} \mathrm{C}$ apartir de ${ }^{14} \mathrm{~N}$. (b) Gráfico de decaimento de ${ }^{14} \mathrm{C}$ em \%/ano (Nave 2005) 
período os nuclídeos cosmogênicos de hélio $\left({ }^{3} \mathrm{He}\right)$, argônio $\left({ }^{37} \mathrm{Ar}\right)$ e a atividade de ${ }^{39} \mathrm{Ar}$ foram medidos (Forman et al. 1971).

Passados 30 anos do trabalho pioneiro de Davis \& Schaffer (1955), embora a técnica se encontrasse empregada em pesquisas em diversos ramos geocientíficos, a datação de superfícies expostas com nuclídeos cosmogênicos ainda não havia adquirido uma rotina laboratorial. Somente em meados dos anos 1980 que essa limitação foi superada com o desenvolvimento da Espectrometria por Aceleração de Massa (Accelerator Mass Spectrometry - MAS), a qual propiciou um crescimento exponencial das pesquisas sobre nuclídeos cosmogênicos, e consequentemente, o início de sua popularização. Os novos equipamentos possibilitaram realizar medidas de razões isotópicas menores que $10^{-15}$ e erros analíticos inferiores a 3\%. Após as novas medidas de ${ }^{14} \mathrm{C}$ (Nelson et al. 1977) por espectrômetros, houve motivação suficiente no meio científico para desenvolver rotinas para o ${ }^{10} \mathrm{Be},{ }^{26} \mathrm{Al}$, etc. Os sucessos nas detecções e as novas aplicações surgidas conduziram aos estudos de nuclídeos cosmogênicos in situ.

Nishiizumi et al. (1989), foram os primeiros a determinar empiricamente a taxa de produção dos nuclídeos cosmogênicos de ${ }^{10} \mathrm{Be} \mathrm{e}{ }^{26} \mathrm{Al}$ em quartzo. Eles utilizaram o quartzo devido a sua baixa concentração de alumínio, que permite a medição de ${ }^{26} \mathrm{Al}$ após exposições tão baixas quanto 103 anos. A integridade do quartzo minimiza a possibilidade de contaminação por chuva com ${ }^{10} \mathrm{Be}$ produzida na atmosfera. E, finalmente, sua ubiquidade garante que ele seja encontrado em uma ampla variedade de configurações geológicas. Esta pesquisa trouxe subsídio para vários estudos posteriores, incluindo a construção da história de exposição do deserto do Líbano, no oeste do Egito por Klein et al. (1986).

A viabilidade de medir quantitativamente ${ }^{10} \mathrm{Be} e$ ${ }^{26} \mathrm{Al}$ produzidos in situ por raios cósmicos em quartzo e as possíveis aplicações de desse par para estudar processos de intemperismo/erosões continentais possibilitou determinar o registro contínuo das mudanças de taxa de erosão através do tempo, além de datações de soterramento (Laureano et al. 2014).

Os isótopos de meia-vida longa, tal como ${ }^{10} \mathrm{Be}$ e ${ }^{26} \mathrm{Al}$, e os gases nobres são especialmente suscetíveis a quantificar as taxas de denudação (Cockburn \& Summerfield 2004). Quantificar estas taxas de denudação, tanto do presente quanto do passado, é uma importante forma de compreensão da evolução geomorfológica de uma região ou bacia. Infelizmente, a interação entre clima, tectônica e geomorfologia ainda é complexa e, por vezes, difícil de ser integrada. Contudo, os nuclídeos cosmogênicos medidos em depósitos sedimentares de idade conhecida como, por exemplo, uma sequência de terraços fluviais, oferecem a possibilidade de determinar suas taxas de denudação. Por outro lado, morfologias com intemperismo tênue podem ser investigadas com um nuclídeos cosmogênico ou mesmo com uma combinação de variados isótopos (Balco \& Stone 2005). Em comparação com os dados de um único isótopo, um número mais expressivo de informações sobre as taxas de denudação pode ser obtido com a utilização de múltiplos isótopos. Neste caso, considerações sobre a mineralogia da rocha pode vir a limitar estas possíveis combinações.

A utilização de múltiplos isótopos é essencial para se distinguir um estágio de exposição simples de um complexo. Baseado na meia-vida de cada isótopo é possível calcular a média de um determinado intervalo de tempo. Os isótopos com meia-vida longa ou estável preservam um número maior de informações e podem ser utilizados no cálculo de taxas de denudação de centenas de milhões de anos. Já isótopos com meia-vida curta, como o ${ }^{14} \mathrm{C}$, são utilizados para investigações de taxas de intemperismo dos últimos 15 mil anos (Dunai \& Lifton 2014).

$\mathrm{O}{ }^{36} \mathrm{Cl}$ é geralmente formado próximo da superfície, principalmente nos primeiros metros das rochas expostas (Schlagenhauf et al. 2010). Sua meia-vida o torna susceptível para ser utilizado em datações com variação entre $60 \mathrm{mil}$ anos e 1,0 Ma (Fig. 5), diferente do nuclídeo cosmogênico de ${ }^{3} \mathrm{He}$, que é medido em minerais. A medição de ${ }^{36} \mathrm{Cl}$ pode ser feita em amostras de rocha total. O método de análise do cloro também pode ser aplicado em área com cobertura glacial e no estudo de águas (Nishiizumi et al. 1989; Phillips et al. 1986). Outro aspecto

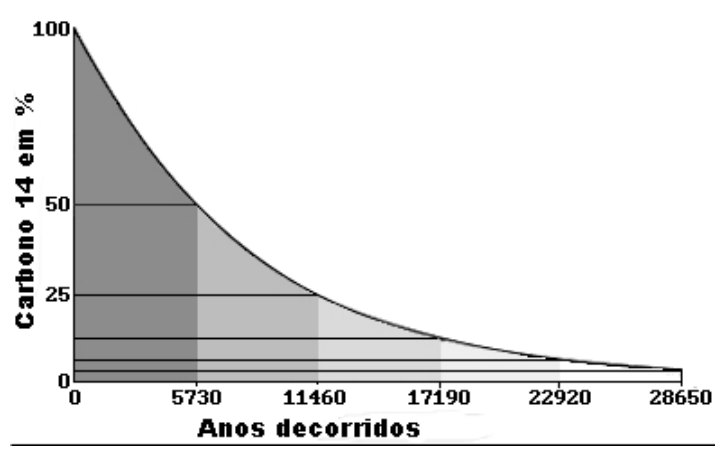

Figura 5. Produção de ${ }^{36} \mathrm{Cl}$ (Schlagenhauf et al. 2010)

\begin{tabular}{c|c|c|c|c|c}
\hline (c) Terrae Didat. & Campinas, SP & v.14 & n.3 & p. 207-216 & jul./set. 2018 \\
\hline \multicolumn{2}{|c|}{210}
\end{tabular}


importante deste método é que a razão ${ }^{36} \mathrm{Cl} /{ }^{35} \mathrm{Cl}$ pode ser utilizada para estimar os conteúdos de urânio na formação de reservatórios. Isto é devido ao acúmulo de cloro em subsuperfície derivadas principalmente em função da concentração de $U$ e Th (Birkle 2006). Embora, Ivy-Ochs et al. (2007) tenham sugerido a medição deste radionuclídeo em sanidina, como demonstrado em seus estudos nos ignimbritos do Chile. O método de análise do cloro também pode ser aplicado a gelo e água (Nishiizumi et al. 1989; Phillips et al. 1986).

As pesquisas em torno de nuclídeos cosmogênicos continuaram, não somente com o objetivo de obterem-se histórias de exposição, mas no aperfeiçoamento das estratégias de amostragem, nos procedimentos laboratoriais das amostras, nas análises e nos aspectos físicos do modelamento dos dados. Vale lembrar que os estudos de nuclídeos cosmogênicos terrestres in situ começaram duas décadas após os estudos de cosmogênicos em meteoritos (in situ), principalmente devido à ausência de parâmetros comparativos e tecnologia adequada para o estudo em rochas.

Concomitante aos estudos sobre ${ }^{10} \mathrm{Be},{ }^{26} \mathrm{Al}$ e ${ }^{36} \mathrm{Cl}$, ocorriam propostas de que o ${ }^{3} \mathrm{He}$ com sua relativa alta taxa de produção cosmogênica e estabilidade nuclear poderia ser medido com um espectrômetro de massa convencional e então posteriormente ser utilizado para determinar idades de exposição (Kurz et al. 1990; Craig \& Poreda 1986). O isótopo estável de ${ }^{3} \mathrm{He}$ é medido em minerais tais como, olivina e piroxênio ricos em $\mathrm{Mg}$, ou apatita, zircão, granada e óxidos de ferro e titânio (Gosse \& Phillips 2001; Ackert et al. 2003; Williams et al. 2005; Licciardi et al. 2006; Dunai et al. 2007).

Williams et al. (2005) mostraram que o clinopiroxênio também é um forte candidato para a medição de ${ }^{3} \mathrm{He}$. Em rochas basálticas, com idade em torno de 3,0 Ma, o clinopiroxênio tem baixas concentrações de hélio magmático. Isto torna a determinação das concentrações de hélio cosmogênico relativamente simples, pois além deste isótopo só há a presença do hélio radiogênico. Este estudo abriu um novo espectro no âmbito do potencial de investigação relacionado à evolução geomorfológica de rochas antigas, desde que tenham histórias de exposição curtas.

$\mathrm{O}^{21} \mathrm{Ne}$ é geralmente utilizado para determinar as idades de exposição de superfícies, as taxas de erosão, as taxas de produção e o transporte de sedimentos. $\mathrm{O}^{21} \mathrm{Ne}$ tem importantes aplicações adicionais, pois, como ${ }^{10} \mathrm{Be} \mathrm{e}{ }^{26} \mathrm{Al}$, é medido no quartzo
(Balco \& Shuster 2009). Estes isótopos partilham os mesmos objetivos e mecanismos de produção, mas têm diferentes meia-vida $\left({ }^{21} \mathrm{Ne}\right.$ é estável, o ${ }^{26} \mathrm{Al}$ $=1,36 \mathrm{Ma} \mathrm{e},{ }^{10} \mathrm{Be}=0,7 \mathrm{Ma}$ ). Assim, combinado com os isótopos cosmogênicos de ${ }^{26} \mathrm{Al}$ e ${ }^{10} \mathrm{Be}$, ele pode ser utilizado para quantificar as histórias de exposição complexas (Klein et al. 1986; Lal 1991) e datar sedimentos ricos em quartzo (Granger \& Riebe 2014).

Algumas questões importantes relacionadas a formação de reservatórios de óleo puderam ser esclarecidas devido ao contínuo crescimento das investigações em torno de nuclídeos cosmogênicos e suas aplicabilidades. A formação de hidrocarbonetos, o tempo de maturação de hidrocarbonetos e a direção da migração de hidrocarbonetos foram então estudadas a partir da medição de ${ }^{129} \mathrm{I}$ (Birkle 2006). Embora, o iodo tenha um comportamento conservador nas águas, a sua forte associação com a matéria orgânica o torna geoquimicamente diferente de outros traçadores isotópicos.

Ambicionando a abordar e discutir estas questões, nos últimos anos vários ensaios e compilações foram propostas e publicadas (Gossi et al. 2001; Grange \& Riebe 2014; Desilets et al. 2006; Licciardi et al. 2006; Balco \& Rovey 2008; Balco \& Shuster 2009). Estes estudos demonstraram que os nuclídeos cosmogênicos vem se tornando uma ferramenta auxiliar no âmbito de pesquisas geológicas e geocientíficas (Tab. 1; Fig. 6).

Consequentemente, várias tentativas vêm sendo feitas para que sejam utilizados outros isótopos cosmogênicos, bem como outros minerais. Por exemplo, os nuclídeos cosmogênicos de $\mathrm{Mn}$ e $\mathrm{Na}$ e, os minerais de granada (Dunai \& Roselieb 1996) e sanidina (Kober et al. 2005; Ivy-Ochs et al. 2007).

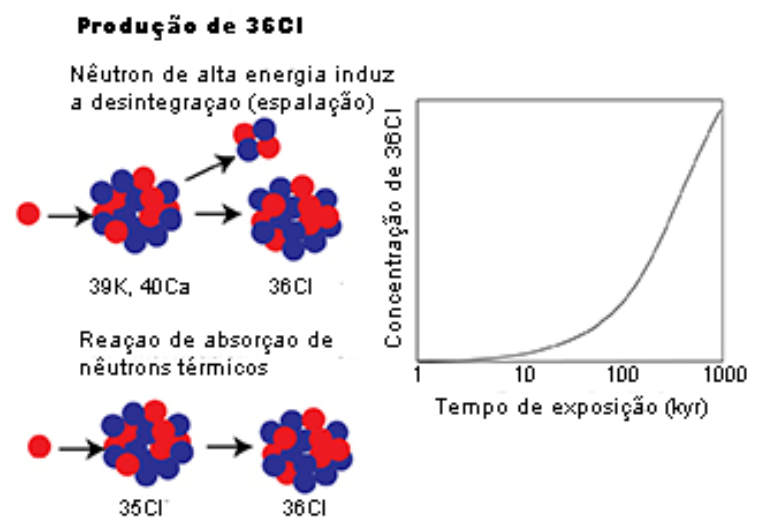

Figura 6. Propriedades como meia-vida e principais minerais de ocorrência dos seis nuclídeos cosmogênicos mais comuns (Darvill 2013) 
Por fim, os dados de nuclídeos cosmogênicos podem ser combinados com as técnicas de termocronologia de baixa temperatura, tais como: Traços de Fissão, e (U-Th)/He, os quais revelam taxas de denudação na casa dos milhões de anos (Schaller \& Ehlers 2006).

Assim, a erudição dominada pela ciência até o presente momento entorno destas técnicas e, principalmente, acerca dos elementos químicos aos quais estas técnicas se aplicam, apresenta-se desenvolvida em diferentes - e singulares - estágios de maturidade. Deste modo, o estado da arte mais consolidado no que diz respeito à produção, acumulação ou decaimento dos isótopos de hélio, berílio, carbono, neônio, alumínio e cloro $\left({ }^{3} \mathrm{He},{ }^{10} \mathrm{Be},{ }^{14} \mathrm{C},{ }^{21} \mathrm{Ne},{ }^{26} \mathrm{Al}\right.$ e ${ }^{36} \mathrm{Cl}$ ) (Gosse \& Phillips 2001) ainda determinam sua mais ampla aplicação e aceitabilidade na pesquisa geocientífica (Laureano et al. 2014).

\section{Princípio Físico}

\section{Taxas de produção}

As taxas de produção de isótopos cosmogênicos em rochas e minerais na superfície da Terra, foram estimadas de acordo com três métodos: espalhamento; captura neutrônica; e muons. A taxa de produção dos isótopos cosmogênicos é uma função entre a profundidade da rocha e a sua elevação (Lal 1991; Lal \& Peters 1967). Em função disto, a grande maioria dos nuclídeos cosmogênicos, cerca de 98\% deles, são gerados nos níveis mais superiores da superfície terrestre, por meio de reações de espalhamento de nêutrons. Enquanto que uma ínfima porcentagem dos raios cósmicos é composta por múons, e por isso, apresentam energia mais elevada, sendo assim, capazes de produzir nuclídeos cosmogênicos em profundidades relativamente maiores (Dunai \& Wiibrans 2001). Isto se dá porque dois agentes principais influenciam o fluxo de nêutrons: o primeiro é o campo magnético da Terra e o segundo é a atmosfera terrestre. Os prótons primários facilmente penetram o campo magnético nos pólos onde as linhas de campo fazem um ângulo conhecido com a superfície da Terra. Desta forma, as taxas de produção são mais altas nos pólos e mais baixas na linha do Equador.

Nos últimos 30 anos, alguns estudos (Zreda et al. 1991; Dunai \& Wijbrans 2000; Jackson et al. 2004) foram realizados com o objetivo de calibrar as taxas de produção para diversas localidades geográficas e, também, de feições geológicas.
Para situações onde está claro que as superfícies apresentam uma história simples de exposição de única fase, ou seja, nenhuma erosão ou soterramento como primeira exposição, a concentração de nuclídeos cosmogênicos à superfície, $\mathrm{N}(0)$, acumula como uma função de tempo de acordo com Lal (1991) e pode ser determinada a partir de:

$$
N(0)=\frac{P(0)}{\lambda}\left(1-e^{-\lambda T}\right)
$$

Para radionuclídeos, e para nuclídeos estáveis pode ser representada como se segue:

$$
N(0)=P(0) T N(0)=P(0) T
$$

Nesta equação, $P(0)$ é a taxa de produção na superfície do nuclídeo cosmogênico; $\lambda$ é a constante de decaimento radioativo, e $T$ é o tempo de exposição.

O limite superior de uma idade de exposição depende do nuclídeo utilizado. No caso de radionuclídeos em uma superfície sem perturbações, o limite é determinado quando ocorre um equilíbrio secular, que acontece em quatro ou cinco meias-vidas.

O comprimento curto de atenuação para a produção de nuclídeos cosmogênicos afeta a quantidade das taxas de erosão ou soterramento de uma superfície, embora possa ser tolerada sem perda na precisão dos dados. O contínuo surgimento de novas superfícies por erosão descobre o material anteriormente coberto. Superfícies erodidas têm concentrações de nuclídeos cosmogênicos mais baixas que superfícies bem conservadas de mesma.

Neste caso, a superfície erodida é considerada. Isto pode ser demonstrado pela equação 3 :

$N(0)=\left[P_{n}(0) /\left(\lambda+\rho \epsilon / L_{0}\right)\right]\left(1-e^{-\left(\lambda+\rho \epsilon / L_{0}\right) T}\right)+N_{i n h} e^{-\lambda T}$

Nesta equação, $\operatorname{Pn}(0)$ é a taxa de produção do nuclídeo cosmogênico; $\lambda$ é a constante de decaimento radioativo; $\rho$ é densidade da rocha; $\epsilon$ é a taxa de erosão; $\mathrm{L}_{\mathrm{o}}$ é o comprimento de atenuação na rocha; T é o tempo de exposição; $\mathrm{N}_{i n h}$ é a concentração do nuclídeo cosmogênico durante a exposição inicial.

$\mathrm{Na}$ equação 3 , a dependência da taxa de produção de nuclídeos cosmogênicos em relação à profundidade segue uma lei exponencial simples. Entretanto, em situações onde as superfícies têm taxas de erosão entre $10 \mathrm{~m} / \mathrm{Ma}$ e $1 \mathrm{~m} / \mathrm{Ma}$, ou onde o soterramento não é suficientemente espesso o 
desprezo das taxas de produção de muons podem conduzir a erros significantes no modelamento das taxas de erosão ou no cálculo de tempos do soterramento (Granger \& Muzikar 2001), as taxas de erosão entre o limite superior e inferior necessitam de uma equação mais completa, que incorpore as taxas de captura neutrônica em conjunto com muons (Granger \& Muzikar 2001), como demonstrado pela Equação 3.

Alguns isótopos cosmogênicos como $\mathrm{o}^{36} \mathrm{Cl}$, por exemplo, apresentam uma taxa de produção que aumenta com a latitude devido a uma diminuição no campo magnético da terra, e aumenta também com a altitude devido a atenuação dos raios cósmicos na atmosfera (Lal \& Peters 1967; Lal 1991). Como consequência disto, as taxas de produção têm que ser adaptadas de acordo com a longitude, a latitude e a altitude do local de amostragem.

A quantidade de ${ }^{36} \mathrm{Cl}$ cosmogênico acumulado em uma dada amostra depois de $t$ anos de exposição a raios cósmicos e com taxas de erosão desprezíveis pode ser expressa pela equação a seguir:

$R-R_{0}=\frac{E_{n} L_{n} D_{n}\left(\psi_{K} C_{K}+\psi_{C a} C_{C a}+\psi_{n}\right)+E_{\mu} L_{\mu} \psi_{\mu}-\left(1-e^{-\lambda t}\right)}{\lambda N}$

Nesta equação, $\mathrm{R}=$ razão ${ }^{36} \mathrm{Cl} / \mathrm{Cl} ; R 0=$ background da razão ${ }^{36} \mathrm{Cl} / \mathrm{Cl} ; \psi_{K} C_{K}=$ taxas de produções devido ao espalhamento do ${ }^{39} \mathrm{~K}$ e ${ }^{40} \mathrm{Ca} ; C_{K} C_{a}=$ concentrações de $\mathrm{K}$ ou $\mathrm{Ca} ; \psi n=$ taxa de produção devido a ativação neutrônica do ${ }^{35} \mathrm{Cl} ; \psi_{\mu}=$ taxa de produção devido a captura de muons do ${ }^{40} \mathrm{Ca} ; \mathrm{n}=$ nêutrons; $\mu=$ muons; $\mathrm{E}=$ altitude; $\mathrm{L}=$ latitude; $\mathrm{D}=$ profundidade; $t=$ tempo de exposição; $N$ $=$ concentração do $\mathrm{Cl}$ estável e $\lambda=$ constante de decaimento para $\mathrm{O}^{36} \mathrm{Cl}$.

Os fatores de escala para os nêutrons ao nível do mar $\left(E_{\mathrm{n}}\right)$ e para as latitudes $\left(L_{\mathrm{n}}\right)$ podem ser calculados (Lal \& Peters 1967; Lal 1991) a partir de uma exponencial simples $\left(-\mathrm{d} / \Lambda_{\mathrm{n}}\right)$, onde $\Lambda_{\mathrm{n}}$ corresponde à média do percurso livre de nêutrons (Lal 1991). Um maior detalhamento para os cálculos pode ser observado em Zreda et al. (1991).

O conteúdo de ${ }^{3} \mathrm{He}$ cosmogênico é calculado a partir da diferença entre o ${ }^{3} \mathrm{He}$ da amostra e o ${ }^{3} \mathrm{He}$ magmático, como demonstrado pela equação a seguir (Kurz et al. 1990):

$$
{ }^{3} \mathrm{He}_{\text {cosmogênico }}={ }^{3} \mathrm{He}_{\text {total }} \mathrm{x}{ }^{3} \mathrm{He}_{\text {mantélico }}
$$

A quantificação do ${ }^{3} \mathrm{He}$ mantélico pode ser obtida através da medida da razão ${ }^{3} \mathrm{He} /{ }^{4} \mathrm{He}$ após moagem a vácuo dos grãos de olivina, e o total de ${ }^{4} \mathrm{He}$ na amostra, como demonstrado pela equação utilizada por Licciardi et al. (2006):

$$
{ }^{3} \mathrm{He}_{\text {mantélico }}=\left(\left[{ }^{3} \mathrm{He} /{ }^{4} \mathrm{He}\right]_{\text {mantélico }}\right) \times{ }^{4} \mathrm{He}_{\text {total }}
$$

Durante muitos anos o estudo de Lal (1991) foi o padrão utilizado para os fatores de correção para latitude e altitude, mas estudos mais modernos como os de Dunai \& Wiibrans (2000) e Desilets et al. (2006) introduziram novos modelos a serem seguidos. Estas pesquisas utilizam novas aproximações, parcialmente fundamentadas em novos dados de fluxo de nêutrons. Comumente, são utilizados os fatores de escala inicialmente descritos por Lal (1991).

Em pesquisas com isótopos cosmogênicos, um importante aspecto é o decréscimo das incertezas associadas com as taxas de produção, bem como com os fatores de latitude e altitude. Estes avanços ajudam na diminuição do erro analítico das idades de exposição e taxas de denudação determinadas com a precisão do espectrômetro de massa com acelerador e dos espectrômetros de massa para gases nobres. Atualmente, o erro varia de 1 a $4 \%$.

\section{Idade de exposição}

A idade de exposição de uma superfície é a medida direta da duração do tempo de exposição da rocha. Esta concentração pode ser expressa pela clássica equação de Lal (1991):

$$
\begin{aligned}
& N=\frac{p}{\frac{p E}{\Lambda}}\left(1-e^{-\left(\frac{\rho \epsilon}{\Lambda}\right) T}\right) \\
& N=\frac{p}{\frac{p E}{\Lambda}}\left(1-e^{-\left(\frac{\rho \epsilon}{\Lambda}\right) T}\right)
\end{aligned}
$$

Nesta equação, $\mathrm{N}\left(\right.$ at $\left.^{-1}\right)=$ concentração do nuclídeo radiogênico; $\mathrm{P}\left(\right.$ at g$\left.^{-1} \operatorname{anos}^{-1}\right)=$ taxa de produção do nuclídeo radiogênico; $\mathrm{T}$ (anos) $=$ tempo de exposição; $\rho\left(\mathrm{g} \mathrm{cm}^{-3}\right)=$ densidade da rocha; $C\left(\mathrm{~cm} \mathrm{anos}^{-1}\right)=$ taxa de erosão; $\Lambda\left(\mathrm{g} \mathrm{cm}^{-2}\right)$ $=$ comprimento da atenuação do raio cósmico; $\lambda$ $\left(\mathrm{a}^{-1}\right)=$ constante de decaimento do radionuclídeo.

No caso de radionuclídeos, o tempo de acumulação é inversamente proporcional a quantidade do decaimento radioativo, o que proporciona seu cálculo a partir da equação a seguir: 


$$
\begin{aligned}
& N_{0}=\frac{P}{\lambda+\frac{\rho \epsilon}{\Lambda}}\left(1-e^{-\left(\lambda+\frac{\rho \epsilon}{\Lambda}\right) T}\right)+N e^{-\lambda T} \\
& N_{0}=\frac{p}{\lambda+\frac{\rho \epsilon}{\Lambda}}\left(1-e^{-\left(\lambda+\frac{\rho \epsilon}{\Lambda}\right) T}\right)+N e^{-\lambda T}
\end{aligned}
$$

Nesta equação, $\lambda\left(\mathrm{a}^{-1}\right)=$ constante de decaimento do radionuclídeo.

Alguns destes parâmetros são conhecidos e amplamente utilizados, tal como a constante de decaimento de um radionuclídeo (lei Geiger-Nuttal), ou a densidade da rocha (Balco \& Stone 2005), ou ainda o comprimento da atenuação do raio cósmico. Outras variáveis são obtidas a partir da análise da amostra no espectrômetro de massa com acelerador. Um exemplo é a concentração do nuclídeo de interesse. De qualquer forma, talvez o mais importante de tudo seja a exatidão da taxa de produção do nuclídeo alvo, pois dela depende a precisão de uma idade de exposição.

Os modelos utilizados para tal fim são baseados no fluxo de partículas da atmosfera terrestre e nas reações entre nuclídeos, obtidas de várias irradiações experimentais (Leya et al. 2000). Os modelos têm como base a taxa de produção absoluta dos nuclídeos alvo baseada na quantificação de grãos de quartzo, o que proporcionada uma razão. Desta forma, o modelo da taxa de produção elementar é embasado na taxa de produção do $\mathrm{SiO}_{2}$ ou do $\mathrm{Si}$, que pode ser convertida para as taxas equivalentes de olivina e sanidina, usando a medida da composição química destes minerais, por exemplo.

A taxa teórica (Kober et al. 2005) pode ser expressa pela razão $\mathrm{X} / \mathrm{Si}$. Onde, $[\mathrm{X}]$ contém $\mathrm{O}$, $\mathrm{Mg}, \mathrm{Al}, \mathrm{Fe}$ e Ni para o ${ }^{3} \mathrm{He}$, ou [X] contém $\mathrm{O}, \mathrm{N}$, $\mathrm{Mg}, \mathrm{Al}, \mathrm{Ca}$ e Ni. Vale lembrar que estas relações são constantes a todas as latitudes e altitudes até 6000 $\mathrm{m}$, porque a razão $[\mathrm{X}] /[\mathrm{Si}]$ pode variar consideravelmente a altitudes acima de $6000 \mathrm{~m}$.

\section{Considerações Finais}

Este trabalho discute o potencial do emergente campo de nuclídeos cosmogênicos, os mecanismos de produção e as diversas taxas. A técnica de espectrometria de massa com acelerador impulsionou as pesquisas em torno desses isótopos e seus avanços desenvolveram inúmeras aplicabilidades. Atualmente existe plena necessidade de conhecimento no que diz respeito ao aquecimento, soerguimento, subsidência, tectônica e erosão de bacias sedimentares. Conceitos como exumação, soterramento e denudação também são habitualmente utilizados em pesquisas geológicas com o mesmo objetivo; são o resultado de fatores pós-orogênicos, movimentos tectônicos, atividade de hot-spot, mudança eustática do nível do mar, glaciação e acomodação isostática. Assim, as pesquisas envolvendo nuclídeos cosmogênicos proporcionam conhecimentos e descobertas em campos em que não é possível aplicar outros métodos.

Os isótopos estáveis de ${ }^{3} \mathrm{He}$ e ${ }^{21} \mathrm{Ne}$ mostram-se eficientes para os estudos de erosão e outros processos geofísicos, como taxas de soerguimento, subsidência, glaciares e idades de erupções. Alternativamente, são isótopos úteis para caracterizar múltiplas histórias de exposição. Os isótopos cosmogênicos de ${ }^{10} \mathrm{Be},{ }^{26} \mathrm{Al} \mathrm{e}{ }^{36} \mathrm{Cl}$ têm sido utilizados para datar com acurácia a geração de sedimentos, o transporte e as taxas de denudação; quando são integrados a modelos interpretativos os resultados podem ser aplicados para determinar taxas de variações geomorfológicas, como por exemplo, soerguimento e subsidência, ao longo de uma escala que abrange $10^{3}$ a $10^{6}$ anos.

A datação pelo método do isótopo cosmogênico de ${ }^{129}$ I é utilizada para reconstruir o tempo de expulsão do petróleo bruto a partir da rocha-fonte e sua migração até o reservatório atual. $\mathrm{O}{ }^{14} \mathrm{C}$ é o mais conhecido e estudado isótopo cosmogênico, amplamente utilizado em estudos arqueológicos e geológicos para amostras com até 4,0 × $10^{4}$ anos. A alta precisão dos dados gerados com esta técnica proporciona o cálculo das taxas de circulação oceânica global, incluindo o tempo em que uma determinada massa d'água teve contato com a atmosfera. $\mathrm{O}^{39} \mathrm{Ar},{ }^{32} \mathrm{Si},{ }^{81} \mathrm{Kr}$ e o ${ }^{41} \mathrm{Ca}$ cosmogênicos podem ser utilizados para datar a formação do gelo e corpos d'água. Contudo, desde que o método do ${ }^{41} \mathrm{Ca}$ cosmogênico foi proposto em 1979 até os dias de hoje ainda não foi desenvolvido um uso prático.

\section{Referências.}

Ackert R.P., Singes B.S., Guillou H., Kaplan M.R., Kurz M D. 2003. Long-term cosmogenic ${ }^{3} \mathrm{He}$ production rates from ${ }^{40} \mathrm{Ar} /{ }^{39} \mathrm{Ar}$ and $\mathrm{K}-\mathrm{Ar}$ dated Patagonian lava flows at $47 \mathrm{~S}$. Earth Planet. Sci. Lett., 210(1):119-136. http://dx.doi.org/10.1016/S0012821X(03)00134-1.

Anderson E.C., Libby W.F., Weinhouse S., Reid A.F., Kirshenbaum A.D., Grosse A.V. 1947. Natural radiocarbon from cosmic radiation. Phys. Rev., 72(10):931. http://dx.doi.org/10.1103/PhysRev.72.931. 
Arnold J.R., Libby W.F. 1949. Age determinations by radiocarbon content: checks with samples of known age. Science, 110(2869):678-680. http://dx.doi. org/10.1126/science.110.2869.678 .

Balco G., Rovey C.W.I.I. 2008. An isochron method for cosmogenic-nuclide dating of buried soils. Am. J. Sci., 308:1083-1114. http://dx.doi. org/10.2475/10.2008.02 .

Balco G., Shuster D.L. 2009. Production rate of cosmogenic ${ }^{21} \mathrm{Ne}$ in quartz estimated from ${ }^{10} \mathrm{Be},{ }^{26} \mathrm{Al}$ and ${ }^{21} \mathrm{Ne}$ concentrations in slowly eroding Antarctic bedrock surfaces. Earth Planet. Sci. Lett., 281:48-58. http://dx.doi.org/10.1016/j.epsl.2009.02.006 .

Balco G., Stone J.O.H. 2005. Measuring middle Pleistocene erosion rates with cosmic-ray-produced nuclides in buried alluvial sediment, Fisher Valley, southeastern Utah. Earth Surf. Process Landf., 30:1051-1067. http://dx.doi.org/10.1016/j. epsl.2004.12.013 .

Birkle P. 2006. Application of ${ }^{129} \mathrm{I} /{ }^{127} \mathrm{I}$ to define the source of hydrocarbons of the Pol-Chuc, Abkatún and taratunich-Batab oil reservoirs, Bay of Campeche, southern Mexico. J. Geochem. Explor., 89:15-18. .

Bustamante M.C. 2013. Descoberta dos raios cósmicos ou o problema da ionização do ar atmosférico. Rev. Bras. Ens. Fís., 35(2):2603. http://dx.doi. org/10.1590/S1806-11172013000200030 .

Craig H., Poreda R.J. 1986. Cosmogenic ${ }^{3} \mathrm{He}$ in terrestrial rocks: The summit lavas of Maui. Proc. Natl. Acad. Sci., 83(7):1970-1974.

Cockburn H.A.P., Summerfield M.A. 2004. Geomorphological applications of cosmogenic isotope analysis. Prog. Phys. Geogr., 28(1):1-42. https://doi. org/10.1191/0309133304pp395oa .

Darvill C.M. 2013. Cosmogenic nuclide analysis. Geomorphol. Techn., 4:2.10 .

Davis R., Schaeffer O.A. 1955. Chlorine-36 in nature. Ann. New York Acad. Sci., 62(1):107-121.

Desilets D., Zreda M., Almasi P.F., Elmore D. 2006. Determination of cosmogenic ${ }^{36} \mathrm{Cl}$ in rocks by isotope dilution: innovations, validation and error propagation. Chem. Geol., 233(3):185-195. https://doi. org/10.1016/j.chemgeo.2006.03.001 .

Dunai T.J., Lifton N. 2014. The nuts and bolts of cosmogenic nuclide production. Elements, 10:347-350. https://doi. org/10.2113/gselements.10.5.347 .

Dunai T.J.; Roselieb K. 1996. Sorption and diffusion of helium in garnet: implications for volatile tracing and dating. Earth Planet. Sci. Lett., 139(3-4):411-421. https://doi.org/10.1016/0012-821X(96)00029-5 .

Dunai T.J., Wijbrans J.R. 2000. Long-term cosmogenic $3 \mathrm{He}$ production rates $(152 \mathrm{ka}-1.35 \mathrm{Ma})$ from ${ }^{40} \mathrm{Ar}{ }^{39} \mathrm{Ar}$ dated basalt flows at $29 \mathrm{~N}$ latitude. Earth Planet. Sci. Lett., 176(1):147-156. https://doi. org/10.1016/S0012-821X(99)00308-8 .

Dunai T.J., Stuart F.M., Pik R., Burnard P., Gayer E. 2007. Production of ${ }^{3} \mathrm{He}$ in crustal rocks by cos- mogenic thermal neutrons. Earth Planet. Sci. Lett., 258(1):228-236. https://doi.org/10.1016/j. epsl.2007.03.031 .

Forman M.A., Stoenner R.W., Davis R.Jr. 1971. Cosmic-Ray Gradient Measured by the Argon-37/ Argon-39 Ratio in the Lost City Meteorite, J. Geophys. Res., 76:4109-4120. https://doi.org/10.1029/ JB076i017p04109.

Gosse J.C., Phillips F.M. 2001. Terrestrial in situ cosmogenic nuclides: theory and application. Quat. Sci. Rev., 20:1475-1560.

Granger D.E., Muzikar P.F. 2001. Dating sediment burial with in situ-produced cosmogenic nuclides: theory, techniques, and limitations. Earth Planet. Sci. Lett., 188(1):269-281. https://doi.org/10.1016/ S0012-821X(01)00309-0 .

Granger D.E., Riebe C.S. 2014. Cosmogenic Nuclides in Weathering and Erosion. Earth Syst. Environ. Sci., 7:401-436. https://doi.org/10.1016/B978-0-08095975-7.00514-3. .

Grosse A.V. 1934. An unknown radioactivity. J. Am. Chem. Soc., 56(9):1922-1924. https://doi. org/10.1021/ja01324a503.

Hess V.F. 1912. Observations of the penetrating radiation on seven balloon flights. Physik. Zeitschr., 13:1084-1091.

Ivy-Ochs S., Kerschner H., Schlüchter C. 2007. Cosmogenic nuclides and the dating of Lateglacial and Early Holocene glacier variations: the Alpine perspective. Quat. Inter., 164:53-63. https://doi. org/10.1016/j.quaint.2006.12.008 .

Jackson S.E., Pearson N.J., Griffin W.L., Belousova E.A. 2004. The application of laser ablation-inductively coupled plasma-mass spectrometry to in situ U-Pb zircon geochronology. Chem. Geol., 211(1):47-69. https://doi.org/10.1016/j.chemgeo.2004.06.017. .

Klein J., Giegengack R., Middleton R., Sharma P., Underwood J., Weeks R.A. 1986. Revealing histories of exposure using in situ produced ${ }^{26} \mathrm{Al}$ and ${ }^{10} \mathrm{Be}$ in Libyan desert glass. Radiocarbon, 28:547-555. https://doi.org/10.1017/S0033822200007700 .

Kober F., Ivy-Ochs S., Leya I., Wieler R., Baur H., Magna T. 2005. In situ cosmogenic ${ }^{10} \mathrm{Be}$ and ${ }^{21} \mathrm{Ne}$ in sanidine and in situ cosmogenic 3He in Fe-Ti-oxide minerals. Earth Planet. Sci. Lett., 236:404-418. https://doi.org/10.1016/j.epsl.2005.05.020.

Kurz M.D., Colodner D., Trull T.W., Moore R.B., O`Brien K. 1990. Cosmic ray exposure dating with in situ produced cosmogenic ${ }^{3} \mathrm{He}$ : results from young Hawaiian lava flows. Earth Planet. Sci. Lett., 97(1):177-189. https://doi.org/10.1016/0012821X(90)90107-9.

Lal D., Peters B. 1967. Cosmic ray produced radioactivity on the Earth. Handbuch der Physik, p. 551-612.

Lal D., Goldberg E.D., Koide M. 1960. Cosmic-ray-produced silicon-32 in Nature. Science, 131(3397):332337. https://doi.org/10.1126/science.131.3397.332. 
Lal D. 1991. Cosmic ray labeling of erosion surfaces: in situ nuclide production rates and erosion models. Earth Planet. Sci. Lett., 104(2-4):424-439. https:// doi.org/10.1016/0012-821X(91)90220-C. .

Laureano F.V., Granger D.E., Karmann I., Novello V.F. 2014. Datação de soterramento utilizando os isótopos cosmogênicos ${ }^{10} \mathrm{Be} \mathrm{e}{ }^{26} \mathrm{Al}$ : síntese metodológica e breve revisão de suas aplicações em geociências. Geonomos, 22(2):18-27. http://dx.doi.org/10.18285/ geonomos.v22i2.314.

Leya I., Wieler R., Halliday A.N. 2000. Cosmic-ray production of tungsten isotopes in lunar samples and meteorites and its implications for Hf-W cosmochemistry. Earth Planet. Sci. Lett., 175(1):1-12. http:// dx.doi.org/10.1016/S0012-821X(99)00295-2 .

Libby W.F. 1946. Atmospheric helium three and radiocarbon from cosmic radiation. Phys. Rev., 69(11-12):671, 1946. https://doi.org/10.1103/PhysRev.69.671.2.

Licciardi J.M., Kurz M.D., Curtice J.M. 2006. Cosmogenic ${ }^{3} \mathrm{He}$ production rates from Holocene lava flows in Iceland. Earth Planet. Sci. Lett., 246(3):251264. https://doi.org/10.1016/j.epsl.2006.03.016 .

Locker G.L. 1933. Neutrons from cosmic-ray stosse. Phys. Rev., 44:779-81.

Montgomery C.G., Montgomery D.D. 1939. The Intensity of Neutrons of Thermal Energy in the Atmosphere at Sea Level. Phys. Rev., 56:10-17. https://doi.org/10.1103/PhysRev.56.10 .

Nave C.R. 2005. HyperPhysics. Report, ECE dept. Georgia State University.

Nelson D.E., Korteling R.G., Stott W.R. 1977. Carbon-14: Direct detection at natural concentrations. Science, 198(4316):507-508. https://doi. org/10.1126/science.198.4316.507 .

Nishiizumi K., Winterer, E.L., Kohl J.R., Klein J., Middleton R., Lal D., Arnold J.R. 1989. Cosmic ray production rates of ${ }^{10} \mathrm{Be}$ and ${ }^{26} \mathrm{Al}$ in quartz from glacially polished rocks. J. Geophys. Res., 94:1790717915. https://doi.org/10.1029/JB094iB12p17907 .

Reeves H., Fowler W.A., Hoyle F. 1970. Galactic cosmic ray origin of $\mathrm{Li}, \mathrm{Be}$ and $\mathrm{B}$ in stars. Nature, 226(5247):727-729. https://doi. org/10.1038/226727a0 .

Schaller M., Ehlers T.A. 2006. Limits to quantifying climate driven changes in denudation rates with cosmogenic radionuclides. Earth Planet. Sci. Lett., 248(1):153-167. http://dx.doi.org/10.1016/j. eps1.2006.05.027 .

Gaudemer A.S.Y., Benedetti L., Manighetti I., Palumbo L., Schimmelpfennig I., Finkel R., Pou K. 2010. Using in situ Chlorine-36 cosmonuclide to recover past earthquake histories on limestone normal fault scarps: a reappraisal of methodology and interpretations Geophys. J. Inter. 182(1):36-72. https://doi.org/10.1111/j.1365246X.2010.04622.x .

Stoenner R.W., Lyman W.J., Davis Jr.R. 1970. Cosmicray production of rare-gas radioactivities and tritium in lunar material. Geochim. Cosmochim. Acta, 1:1583-1592.

Von Blanckenburg F., Willenbring J.K. 2014. Cosmogenic Nuclides: Dates and Rates of Earth-Surface Change. Elements, 10(5):341-346. http://doi. org/10.2113/gselements.10.5.341 .

Williams A.J., Stuart F.M., Day S.J., Phillips W.M. 2005. Using pyroxene microphenocrysts to determine cosmogenic ${ }^{3} \mathrm{He}$ concentrations in old volcanic rocks: an example of landscape de-velopment in central Gran Canaria. Quat. Sci. Rev., 24:211-222. https://doi.org/10.1016/j.quascirev.2004.07.004

Zreda M.G., Phillips F.M., Elmore D., Kubik P.W., Sharma P., Dorn R.I. 1991. Cosmogenic chlorine-36 production rates in terrestrial rocks. Earth Planet. Sci. Lett., 105(1-3):94-109. https://doi. org/10.1016/0012-821X(91)90123-Y . 